\title{
Rheumatoid arthritis presenting as rheumatoid meningitis: A case report
}

\author{
Ryan Jessee,, Robert T Keenan \\ Duke University Hospital, United States
}

Received: May 5, 2017

DOI: $10.5430 /$ crim.v4n3p17
Accepted: May 31, $2017 \quad$ Online Published: June 7, 2017

URL: https://doi.org/10.5430/crim.v4n3p17

\begin{abstract}
Rheumatoid meningitis typically presents as a late manifestation of rheumatoid arthritis, and its pathogenesis remains uncertain. We describe a patient with neurological symptoms, subsequently found to have a brain mass of unclear etiology. The findings from imaging and histological analysis led to a diagnosis of rheumatoid meningitis. Surprisingly, this case presented in the absence of a history of arthritis or other systemic signs typically seen in RA. This case highlights the fact that rheumatoid meningitis can be the initial presenting symptom of rheumatologic disease and should be kept in the differential in patients with or without a history of RA.
\end{abstract}

Key Words: Rheumatoid meningitis, Rheumatoid leptomeningitis, Rheumatoid arthritis

\section{INTRODUCTION}

Rheumatoid arthritis (RA) is a chronic inflammatory autoimmune disease that primarily affects the synovium, although extra-articular manifestations are common. ${ }^{[1]}$ Neurological complications from RA are classically secondary to musculoskeletal involvement, such as atlanto-axial subluxation affecting the cervical spine. ${ }^{[1]}$ Rheumatoid meningitis is an uncommon and frequently difficult to diagnose manifestation of RA, capable of presenting with a variety of neurological symptoms. ${ }^{[2]}$ However, rheumatoid meningitis is typically a late manifestation of disease, presenting after the diagnosis of RA has already been established. ${ }^{[2]}$ Thus, we present an atypical presentation of rheumatoid meningitis in a patient without a preexisting history of RA.

\section{Case presentation}

A 68-year-old female presented at an academic medical institution for evaluation of a left frontal brain mass of unknown etiology. The patient's past medical history was significant for hypertension, osteoarthritis, and previous pacemaker insertion for symptomatic sinus bradycardia. The patient's symptoms first began four years prior in 2010, with an episode of acute confusion. The patient went to a local hospital in East Tennessee, where imaging reportedly revealed multiple small ischemic strokes for which aspirin was started. Right-sided weakness was initially present and subsequently improved with physical therapy. Two years later, secondary generalized seizures began occurring and the anti-epileptic medication levetiracetam was initiated. The patient's local neurologist ordered a computed tomography (CT) of the head which revealed a left frontal brain mass of unclear etiology. Magnetic resonance imaging (MRI) could not be performed due to the presence of a permanent pacemaker. Biopsy of the brain mass at her local hospital was read by the pathologist as negative for malignancy but otherwise inconclusive. The slides were unavailable for further review by time of referral

*Correspondence: Ryan Jessee; Email: ryan.jessee@duke.edu; Address: Duke University Hospital, United States. 
to our institution. The patient was continued on levetiracetam with worsening seizures and fatigue. Repeat outside CT imaging revealed an increase in the size of the brain mass which led to a referral to our university hospital.

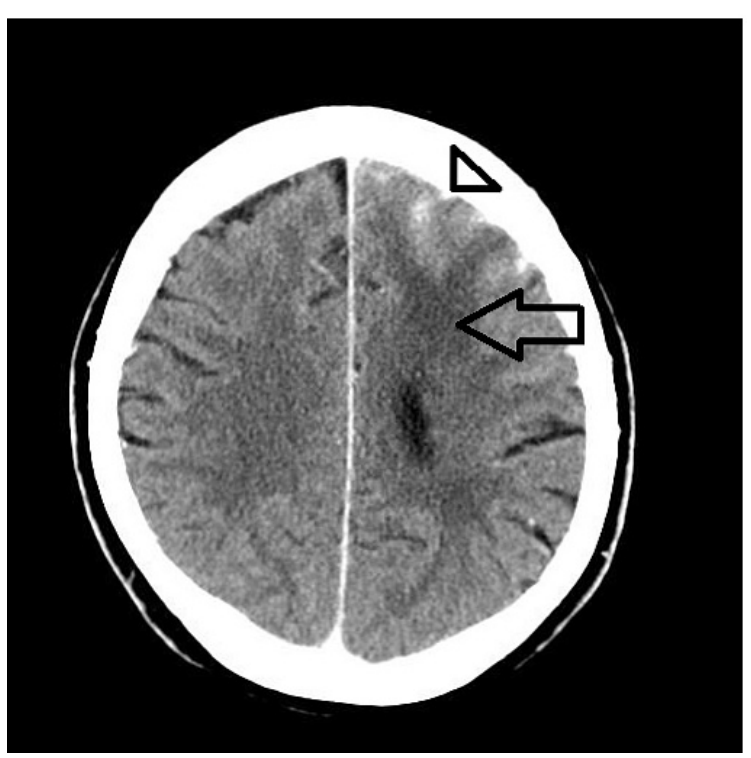

Figure 1. Head CT showed hypoattenuation of white matter in the left frontal lobe (arrow) with leptomeningeal enhancement (arrowhead)

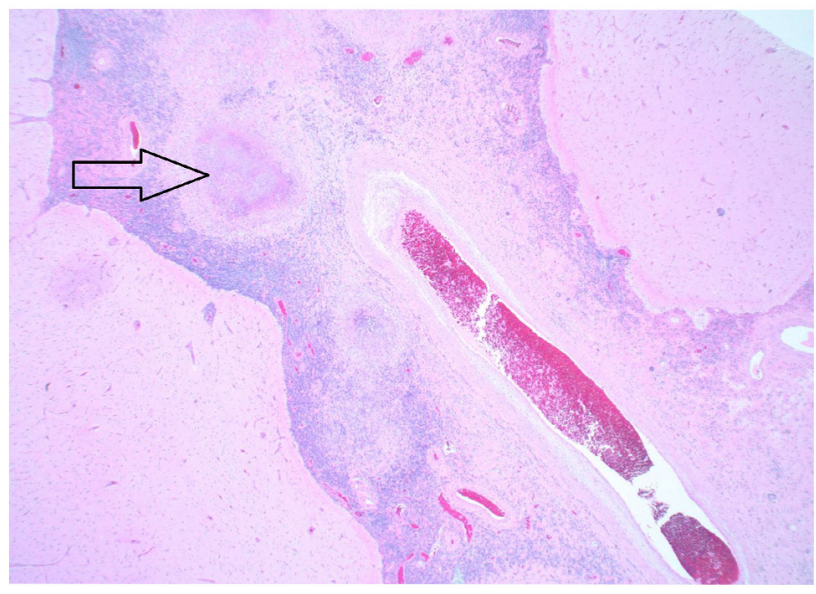

A
On presentation, the patient complained of generalized fatigue, vertiginous symptoms, and worsening right-sided weakness. Head CT showed hypoattenuation of white matter in the left frontal lobe with leptomeningeal enhancement of the overlying sulci (see Figure 1). Cerebrospinal fluid (CSF) studies revealed 8 nucleated cells with 5\% neutrophils, $61 \%$ lymphocytes, and $4 \%$ monocytes. CSF protein was $64 \mathrm{mg} / \mathrm{dl}$, and glucose was $56 \mathrm{mg} / \mathrm{dl}$. Rheumatoid factor (RF) was not tested in the CSF fluid. CSF cytology showed atypical cells with large nucleus to cytoplasmic ratios and abnormal membranes, although flow cytometry was normal. Paraneoplastic panel and neuromyelitis optica antibody testing were negative. Serum protein electrophoresis and IgG levels were within normal limits. CT chest was unrevealing, while cerebral angiogram displayed no angiographic evidence of vasculitis. A biopsy of the brain mass showed necrotizing granulomas in the leptomeningeal space without evidence of malignancy (see Figure 2). No palisading histiocytes or giant cells were seen. No organisms were identified on fungal staining and tuberculosis staining. The official pathology report concluded "the histologic differential diagnosis includes (but is not necessarily limited to) chronic infection, neurosarcoidosis, and autoimmune diseases such as granulomatosis with polyangiitis or rheumatoid arthritis".

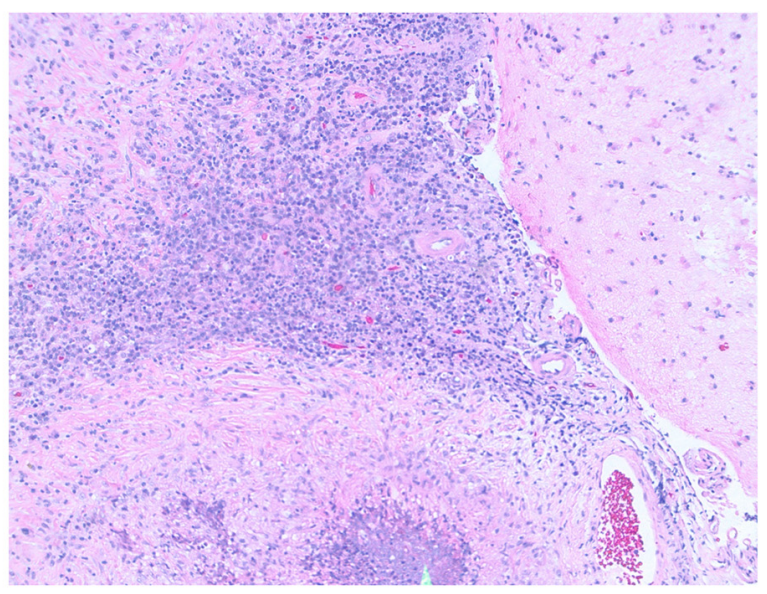

B

Figure 2. Biopsy of the brain mass showed necrotizing granulomas in the leptomeningeal space (arrow) on hematoxylin and eosin stain at $2 \times(\mathrm{A})$ and $10 \times(\mathrm{B})$ magnification

An infectious workup revealed negative CSF gram stain and culture. Other negative CSF studies included toxoplasma polymerase chain reaction (PCR), cytomegalovirus PCR, and VDRL. Serum HIV testing, quantiferon gold, and toxoplasmosis serology were all negative. The brain mass was deemed to be unlikely infectious by infectious disease physicians due to the prolonged time course of disease, negative aforementioned workup, and lack of other infectious symptoms.

Given the unclear cause of the brain mass, a rheumatologic etiology was considered. A complete physical examination revealed no major abnormalities, including no evidence of synovitis detected by a rheumatologist. ANA was positive at a titer of 1: 640, RF was positive at $208(<20 \mathrm{IU} / \mathrm{ml})$, anti- 
CCP antibodies were positive at $95.8(<5.1 \mathrm{U} / \mathrm{ml})$, while antidsDNA and ANCA were negative. Other negative serologies included Anti-Sm, Anti-Ro, Anti-La, and Anti-RNP. Complement levels were normal, and inflammatory markers were not elevated. ACE levels were within normal limits. On further discussion with our pathologists, they felt that the necrotizing granuloma was most consistent with rheumatoid meningitis given the negative infectious workup as well as the extent of necrosis within the granuloma which would be atypical for sarcoidosis.

Our patient was diagnosed with rheumatoid leptomeningitis based on imaging, pathology, serology, and exclusion of other causes. Oral prednisone was started at $40 \mathrm{mg}$ oral daily with improvement in symptoms including decreased seizure episodes and improved fatigue. Methotrexate (MTX) was subsequently initiated given the good response to moderate doses of prednisone, with prednisone slowly weaned over time. On follow-up with her rheumatologist, the patient was noted to have developed inflammatory arthopathy over the intervening months. The patient was subsequently started on infliximab with improvement. Repeat head CT scans at 6 months and 1 year showed stability in the size of the mass, with the patient having no new seizures or neurological symptoms.

\section{DISCUSSION}

Rheumatoid meningitis is a rare neurological complication of RA that can present with a multitude of symptoms, including altered mental status, headaches, cranial nerve deficits, paresis, and strokes. ${ }^{[2-4]}$ As seen in our patient, rheumatoid meningitis can develop irrespective of systemic inflammation and arthritis. ${ }^{[2,5]}$ The rarity of diagnosis limits available information about the disease, with the largest case series using less than two dozen patients. ${ }^{[2,6]}$ Classically this disease presents as a late manifestation of RA; however, there are multiple case reports of the disease presenting in the first year of diagnosis. ${ }^{[2,6]}$ Additionally, we found at least one other case report where rheumatoid meningitis occurred in a patient with no pre-existing history of RA, similar to our patient. ${ }^{[7]}$

The disease affects the pachymeninges, leptomeninges, or both. ${ }^{[2]}$ Regardless of the level of meninges affected, diagnosis and therapy are similar. ${ }^{[2]}$ Serum inflammatory studies typically are of little use, although ferritin and serum IL-2 receptor levels may correlate with meningeal inflammation in some patients. ${ }^{[8]}$ Brain imaging traditionally shows meningeal thickening and localized enhancement. ${ }^{[4]} \mathrm{MRI}$ is the preferred imaging modality, although contrast CT can be used for diagnosis, as seen in our patient who could not un- dergo MRI due to pacemaker placement. ${ }^{[2,9]}$ CSF studies often show elevated protein, with potential CSF biomarkers of disease activity including IL-6 and RF. ${ }^{[2,7,8]}$ CSF studies can occasionally show a pleocytosis and low glucose as well. ${ }^{[7]}$ Typically, histopathological examination of meningeal tissue is required which can reveal rheumatoid nodules, nonspecific meningeal inflammation, or vasculitis. ${ }^{[5]}$ Differential diagnosis for epithelioid granulomas, besides RA, includes meningeal carcinomatosis, neurofibromatosis, lymphoma, Sturge-Weber angiomatosis, syphilis, tuberculosis, sarcoidosis, and other inflammatory disorders. ${ }^{[9]}$ Rheumatoid nodules are not required for diagnosis, and necrotizing granulomas without giant cells or epithelioid cells have previously been seen in rheumatoid meningitis, similar to our own patient. ${ }^{[4]}$ Ultimately, the diagnosis of rheumatoid meningitis is one of exclusion, requiring ruling out more common infectious and inflammatory causes of meningitis.

Treatment for rheumatoid meningitis is based largely on case reports and expert-opinion. ${ }^{[2]}$ Steroids are the mainstay of treatment, often supplemented with adjuvant cyclophosphamide or azathioprine. ${ }^{[2,3,10]}$ The response to steroids can be rapid, with improvement of symptoms in weeks, as seen in our patient. ${ }^{[10]}$ TNF blockers and MTX do not appear to be preventative, and rheumatoid meningitis has been documented to recur when switching from cyclophosphamide to infliximab. ${ }^{[11,12]}$ However, MTX and TNF blockers can be used as a steroid sparing agents for patients who wish to avoid other immunosuppressants such as cyclophosphamide or azathioprine, all of which have previously been used for treatment. ${ }^{[5,12]}$ Additionally, rituximab and tocilizumab have shown promise as a well-tolerated steroid sparing agent. ${ }^{[13,14]}$ Regardless of treatment, rheumatoid meningitis remains a serious and deadly complication of RA with a mortality rate of up to $70 \% .^{[2]}$

Our case highlights the fact that rheumatoid meningitis can present even in the absence of arthritis or other systemic signs typically seen in RA. Rarely, rheumatoid meningitis can be the initial presenting symptom of rheumatologic disease and should be kept in the differential in patients with or without a history of RA. ${ }^{[5,7]}$ Once the diagnosis is suspected, prompt treatment is essential given the poor overall morbidity and mortality rate.

\section{ACKNOWLEDGEMENTS}

We are extremely grateful to Dr. Anne Buckley at Duke University for help with pathological interpretation.

\section{CONFlicts OF InTEREST Disclosure}

The authors have declared no conflicts of interest. 


\section{REFERENCES}

[1] Mouthon L, Dunogue B, Guillevin L. Diagnosis and classification of eosinophilic granulomatosis with polyangiitis (formerly named Churg-Strauss syndrome). Journal of Autoimmunity. 2014; 55 (4849): 99-103. PMid:24530234 https ://doi .org/10.1016/j.ja ut. 2014.01 .018

[2] Greco A, Rizzo MI, De Virgilio A, et al. Churg-Strauss syndrome. Autoimmunity Reviews. 2015; 14(4): 341-8. PMid:25500434 https : //doi.org/10.1016/j.autrev.2014.12.004

[3] Mahr A, Moosig F, Neumann T, et al. Eosinophilic granulomatosis with polyangiitis (Churg-Strauss): Evolutions in classification, etiopathogenesis, assessment and management. Current Opinions in Rheumatology. 2014; 26(1): 16-23. PMid:24257370 https: //doi.org/10.1097/B0R.0000000000000015

[4] Baldini C, Talarico R, Della Rossa A, et al. Clinical manifestations and treatment of Churg-Strauss syndrome. Rheumatic Disease Clinics of North America. 2010; 36(3): 527-43. PMid:20688248 https://doi.org/10.1016/j.rdc.2010.05.003

[5] Khoury P, Grayson PC, Klion AD. Eosinophils in vasculitis: Characteristics and roles in pathogenesis. Nat Rev Rheumatol. 2014; 10(8): 474-83. PMid:25003763 https ://doi .org/10.1038/nrrheum. 2014.98

[6] Nair P, Ochkur SI, Protheroe C, et al. Eosinophil peroxidase in sputum represents a unique biomarker of airway eosinophilia. Allergy. 2013; 68(9): 1177-84. https://doi.org/10.1111/all.12206

[7] Comarmond C, Pagnoux C, Khellaf M, et al. Eosinophilic granulomatosis with polyangiitis (Churg-Strauss): clinical characteristics and long-term follow-up of the 383 patients enrolled in the French Vasculitis Study Group cohort. Arthritis Rheum. 2013 65(1): 270-81. PMid:23044708 https://doi.org/10.1002/art. 37721
[8] West S, Arulkumaran N, Ind PW, et al. Diffuse alveolar hemorrhage in ANCA-associated vasculitis. Intern Med. 2013; 52: 5-13. PMid:23291668 https://doi.org/10.2169/internalmedici ne. 52.8863

[9] Kostianovsky A, Hauser T, Pagnoux C, et al. Alveolar hemorrhage in ANCA-associated vasculitides: 80 patients' features and prognostic factors. Clin Exp Rheumatol. 2012; 30 (1): S77-82. PMid:22640651

[10] Assmann G, Molinger M, Pfreundschuh M, et al. Gastrointestinal perforation due to vasculitis at primary diagnosis of eosinophilic granulomatosis with polyangiitis (EGPA) despite a high dose glucocorticosteroids treatment. SpringerPlus. 2014; 3: 404. PMid:25133090 https://doi .org/10.1186/2193-1801-3-404

[11] Franco DL, Ruff K, Mertz L, et al. Eosinophilic granulomatosis with polyangiitis and diffuse gastrointestinal involvement. Case Reports Gastroenterol. 2014; 8: 329-36. PMid:25473392 https: //doi.org/10.1159/000369129

[12] Rank MA, Ochkur SI, Lewis JC, et al. Nasal and pharyngeal eosinophil peroxidase levels in adults with poorly controlled asthma correlate with sputum eosinophilia. Allergy. 2016; 71(4): 567-70. PMid:26645423 https ://doi.org/10.1111/all.12817

[13] Chaigne B, Terrier B, Theilblemont N, et al. Dividing the Janus vasculitis? Pathophysiology of eosinophilic granulomatosis with polyangiitis. Autoimmun Rev. 2016; 15(2): 139-45. PMid:26506114 https://doi.org/10.1016/j.autrev.2015.10.006

[14] Wechsler ME, Akuthota P, Jayne D, et al. Mepolizumab or placebo for eosinophilic granulomatosis with polyangiitis. N Engl J Med 2017; 376: 1921-32. PMid:28514601 https://doi.org/10.105 6/NEJMoa1702079 